\title{
Application of methods of modeling and forecasting for planning of operating results of fish processing enterprises
}

\author{
Oksana Shikhova ${ }^{1 *}$, Marina Selina ${ }^{1}$, and Vladimir Selin ${ }^{2}$ \\ ${ }^{1}$ Vologda State Dairy Farming Academy, 160555 Molochnoe village, Vologda region, Russia \\ ${ }^{3}$ Director, ART-RYBA LLC, 160014 Vologda, Russia
}

\begin{abstract}
This article deals with the methodological aspects of planning activities of fish-processing companies depending on environmental temperature based on the example of group of companies "ArtFish", Vologda. The method of composing the regression model, which describes daily revenue trend dependence from dynamic of daily environmental temperature as a factor defining the demand for fish and fish products is proposed. The sales revenue forecasts taking into account the impact of daily environmental temperature dynamic and the weekly cycle of fluctuation in sales revenue is received. The results of approbation of this method form the basis of its usage in real conditions during the period of digital economy. This allows us making the informed decision for defining the planning volumes of production output for sales.
\end{abstract}

\section{Introduction}

The production of fish processing enterprises is an important component of human diet [1]. The world practice points to the fact that about $17 \%$ of animal and $7 \%$ of the total consumable protein fall to the share of fish nowadays [2]. However, these products have a definite specificity of storage; it necessitates the planning of production and sales volume [3]. Therefore, a need for search of possible methods of regulation of production volumes of a fish processing enterprise appeared.

Due to the specific peculiarities strategic planning of sectors of agro-industrial complex causes many methodical problems, especially in the conditions of market-oriented economy [4]. Close dependence on accidental natural factors strengthened by the instability of market environment determines the difficulty of production planning of an individual enterprise for the long run [5].

In the sphere of population's consumption of food products the forecasting of consumption volumes and revelation of consumption impact factors play the important role. According to the example of fresh fish processing and selling business we tried to define main factors impacting the population's consumption and sales turnover in this industry not paying attention to inflation fluctuation of prices for above-mentioned goods, trade discounts as well as to consumption increases during holidays and pre-holiday days.

Having the statistic data on daily revenue from company's fish products sales in this field of trading sector we performed the analysis of the dynamic of the revenue indicator during statistically long period of time, starting from July 2016 till June 2019.

According to empirical observations, it was found that during the days with the higher daily average environmental temperature consumer demand for production of fish processing enterprises decreased. This is quite accountably because in the specified weather conditions human heat elimination decreases [6] and incased consumption of products with great number of calories is not needed.

The products of the enterprises under study exactly belong to such products. Therefore, the research was aimed at the study and development of the methods which allowed planning the volumes of production and sales, taking into consideration the forecasting data of daytime environmental temperature on the relevant territory.

As a confirmation, the following hypothesis turns out to be possible in the conditions of the digital economy:

While planning daily, weekly, in general, chronologically repeating procurement activities, it is necessary to take into account the weather factor, firstly temperature regime of environment.

A possible connection between the volume of sales and environmental temperature is especially topical for remote areas, where deliveries are carried out once a week or more rarely, and also for rural areas, where in conditions of spring flood, for example, a possibility of delivery is absent (for example, Shenkursk, Archangelsk region).

It is effective to consider planning weekly, as fish and sea products are not the products of daily consumption. The natural factor can be used for more precise forecasting of income in a retail location. A plan

* Corresponding author: oksana-shikhova@yandex.ru 
should be realistic and should motivate an employee for a rational increase of labor intensity [7].

The purpose of the research is to define and make a model of the dependence of the volume of consumer demand for production of a fish processing enterprise from a temperature regime of an environment and develop methods of its forecasting. During the research the methods of econometric modeling on the basis of time series were used in complex. The methods included the building of trend models, models with a cyclical component and regression models.

The practical implication of the research is in the possibility to plan volumes of production and sales of the products of a fish processing enterprise depending on the natural and climatic factor.

\section{Results}

The research was carried out in several steps because it required studying the character and peculiarities of the dynamics of an index of daily revenue profoundly and preparing the data for modeling.

1. In order to verify the presence of correlation dependence between indexes under study linear correlation coefficients were calculated, thus, the approach considering seasonality (season and month) was taken as a basis of data grouping [8]. The results of the calculations did not prove the presence of statistically important and firm correlation between the indexes in spite of its presence in practice, as it was mentioned above.

2. Further, it was reviewed how the strength of correlation between the time series of the indexes under study changed in dependence to the temperature interval. Since the practical experience of control showed that the biggest elasticity of demand from temperatures could be seen only by its positive values that was why the data for the days when the environmental temperature was above $0{ }^{\circ} \mathrm{C}$ (the period from March till November of each year, the exception was 2017 with quite a warm beginning of December and 2019 with positive temperatures in February). As a result, the series of values of correlation coefficients were received and it proved that with the reduction of the range of values of positive temperatures by the transfer to the high values (summer months of the year) dependence between climatic conditions and customer demand for the production was diminishing.

Maximum correlation is achieved only when covering the entire positive temperature range. Inclusion of days when negative temperatures were observed in the processed data (for example, winter moths) leaded to the decrease of correlation coefficient, which proved the fact of the presence of significant correlation between the studied traits only for positive temperatures.

Basing on the results received for the whole data (period of 632 dates) we built the regression model reflecting correlation of sales of sea products daily revenue from the air temperature:

$$
\tilde{y}_{x}=332215.6604-4020.1884 \cdot x
$$

where $y$ - volume of daily revenue from sales of fish products, rubles; $x$ - daily air temperature.
Testing of this model for statistical significance using Fisher $(F=213)$ and Student $\left(t_{1}=74.9 ; t_{2}=14.6\right)$ criteria showed its reliability with statistical probability not less than 0.95 . The residual analysis of the model showed its occasional and normal distribution pattern and feasibility of homoscedasticity requirements. Basing on this model and given its standard error we calculated interval forecasts of daily revenue for certain temperature meanings.

Nevertheless, despite the statistical significance of the model, which is clearly defined by the wide data range used for the model, its approximating qualities were only satisfactory because the value of medium approximation error was $16,7 \%$, it increases its limit values of 5-7\%. The accuracy of the obtained forecast intervals was insufficient in terms of their practical significance for business planning.

The high value of this error could be explained by the presence of autocorrelation of residues (random deviations) for the given model, since the presence of trends in the time series of the studied characteristics is obvious, which can be eliminated by adding a time factor $\mathrm{t}$ into the regression model. However, a residual analysis revealed a lack of autocorrelation (coefficient value was $0.05)$.

As the main research purpose was to develop practically effective econometric model of daily revenue with the aim of further forecasting using it we decided to reduce sample size to one month period due to the fact that when making decisions based on models, it is necessary to operate with actual information and make forecasts for the nearest future.

3. The studies of economic models of dependence of revenue on temperature by selections of starting data reduced up to the period of one month pointed at their statistic insignificance and low approximate features, at that additional inclusion of time factor into the models did not allow improving statistic characteristics of the received regression equation because of the weak intensity of the trend component in the dynamics series of the dependent variable [9]. These results led to the necessity of more profound study of the structure of time series of values of daily revenue during a month. The hypothesis was formulated that in addition to the trend component, which, as the study showed, has a weak expressiveness (trend almost coincides with the line of average monthly level), the dynamics range has a cyclic component, the presence of which in the processed samples does not allow obtaining statistically significant models with a good approximation of the dependence between the.

4. The analysis of the structure of time series of daily revenue for different months based on the calculation of auto-correlation coefficients of levels depending on a lag (L) showed the presence not only the trend component having linear character but also a weakly cyclical component. As a result, a hypothesis was made that the presence of this cyclical component in the selections used for building of regression models deteriorated their approximate features and required preliminary data correction by means of its exclusion. 
5. The method of moving mean was taken as a basis of estimation of the cyclical component values calculated for the period of 7 days. The data for three weeks were included into selections under study with the forecasting of the received econometric model of levels of daily revenue for the perspective forth week. As it was found out in the process of the research, belonging of a selection of a perspective week to one month did not matter, only their chronological order was important.

6. Initially additive models with the cyclical weekly component were built and tested in relation to approximate feature for different three-week periods:

$$
Y=T+S+E,
$$

where $Y$ - level of time series of daily revenue;

$T$ - trend component - a part of value of revenue level conditioned by the main (tendency) trend;

$S$ - cyclical component - a part of value of revenue level conditioned by the cycle of its weekly fluctuations;

$E$ - random component - a part of value of revenue level conditioned by the influence of random factors.

Afterwards, multiplicative models with the cyclical weekly component were studied with the purpose of search of regression models which were the best regarding approximation:

$$
Y=T \cdot S \cdot E .
$$

7. The approximate features of the receiving models with the cyclical component were studied based on dispersion analysis and calculation of the value of a determination coefficient. In most cases the multiplicative model gave higher determination coefficient than additive one, at the same time the value of this index for both models exceeded 0.7 ; that fact pointed to their quite high approximate features (table 1).

High approximating quality of the resulting models can be seen graphically (figs 1 and 2), showing the actual and aligned dynamics of daily revenue, including the trend and cyclical components.

Table 1. Results of the dispersion analysis of models with a cyclic component for three-week time series of daily revenue for different time periods in 2016-2019

\begin{tabular}{|c|c|c|}
\hline \multirow{2}{*}{$\begin{array}{c}\text { Analyzed period (time } \\
\text { range of 3 weeks) }\end{array}$} & $\begin{array}{c}\text { Determination coefficient } \\
\text { Additive } \\
\text { model } \\
Y=T+S+E\end{array}$ & $\begin{array}{c}\text { Multiplicative } \\
\text { model } \\
Y=T \cdot S \cdot E\end{array}$ \\
\hline $04.07 .2016-24.07 .2016$ & 0.829 & 0.922 \\
\hline $01.08 .2016-21.08 .2016$ & 0.758 & 0.846 \\
\hline $05.09 .2016-25.09 .2016$ & 0.845 & 0.944 \\
\hline $22.07 .2017-11.06 .2017$ & 0.749 & 0.844 \\
\hline $03.07 .2017-23.07 .2017$ & 0.751 & 0.766 \\
\hline $21.08 .2017-10.09 .2017$ & 0.775 & 0.853 \\
\hline $14.05 .2018-03.06 .2018$ & 0.816 & 0.912 \\
\hline $02.07 .2018-22.07 .2018$ & 0.826 & 0.916 \\
\hline $18.03 .2019-07.04 .2019$ & 0.813 & 0.911 \\
\hline $03.06 .2019-23.06 .2016$ & 0.706 & 0.783 \\
\hline
\end{tabular}

8. Further, in the processed selection after the exclusion of values of the cyclical component from the starting levels of the daily revenue counted for each day of the week, analytic equalization of the received time series was made without cyclicality using linear function of the trend. Herewith, based on the previous conclusions exclusion of the cyclical component was accomplished in accordance with the multiplicative model (2) by dividing the starting level of the revenue by the index of the cyclical component corresponding a day of a week.

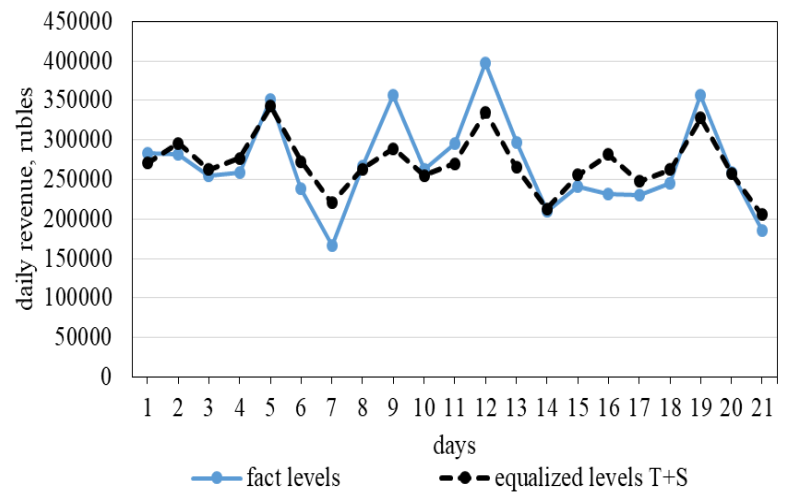

Fig. 1. Actual and equalized, including additive trend and cyclical components $(\mathrm{T}+\mathrm{S})$, dynamics of daily revenue for the period from the $3^{\text {rd }}$ of June 2019 to the $23^{\text {rd }}$ of June 2019

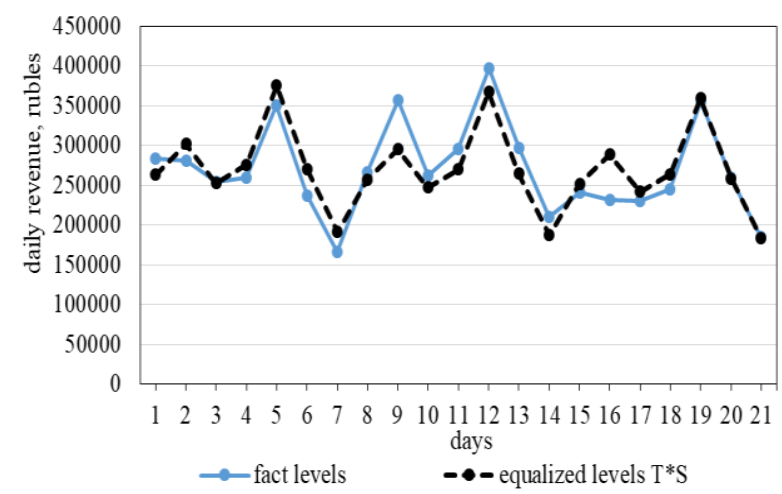

Fig. 2. Actual and equalized, including multiplicative trend and cyclical components $(\mathrm{T} \cdot \mathrm{S})$, dynamics of daily revenue for the period from the $3^{\text {rd }}$ of June 2019 to the $23^{\text {rd }}$ of June 2019

9. The study of presence of correlation between the time series of temperature and revenue for which in one case the trend and cyclical components together were included into the analysis multiplicatively $(\mathrm{T} \cdot \mathrm{S})$, in the other case - only the trend component $(\mathrm{T})$, it showed at a minimum a noticeable and, in most cases of processed selections, an inverse correlation dependence for the time series of revenue having only trend $(\mathrm{T})$. For the time series of revenue $T \cdot S$ a statistically significant correlation was not noticed, it proved a supposition that the presence of the weekly cyclical component did not allow correlation appearing between the time series of the studied features and led to the regression model with the low approximate characters.

The results of the correlation- regression analysis of the dependence between daily environmental temperature and trend of daily revenue according to the selections with periodicity of three weeks formed with the shifting for one date for the period March-April 2019 are presented in the table 2 . 
Table 2. Results of the correlation- regression analysis of the dependence between daily environmental temperature and trend of daily revenue according to the selections formed with the shifting for one date for the period March-April 2019

\begin{tabular}{|c|c|c|c|c|}
\hline \multirow{2}{*}{ Regression model } & Correlation & Fisher's ratio test \\
\cline { 2 - 5 } & $\mathrm{R}$ & $\mathrm{R}^{2}$ & $\mathrm{~F}$ & $\begin{array}{c}\text { Significance } \\
\text { level F }\end{array}$ \\
\hline \multicolumn{5}{|c|}{$18.03 .2019-07.04 .2019$} \\
\hline$\tilde{y}=290044.4-1950.12 \cdot x$ & -0.748 & 0.56 & 24.2 & 0.0001 \\
\hline \multicolumn{5}{|c|}{$19.03 .2019-08.04 .2019$} \\
\hline$\tilde{y}=370048.8-4683.42 \cdot x$ & -0.818 & 0.67 & 38.4 & 0.0000 \\
\hline \multicolumn{5}{|c|}{$20.03 .2019-09.04 .2019$} \\
\hline$\tilde{y}=361563.5-3838.83 \cdot x$ & -0.827 & 0.69 & 41.6 & 0.0000 \\
\hline $21.03 .2019-10.04 .2019$ \\
\hline$\tilde{y}=363697.1-4670.12 \cdot x$ & -0.823 & 0.68 & 39.9 & 0.0000 \\
\hline \multicolumn{5}{|c|}{$22.03 .2019-11.04 .2019$} \\
\hline$\tilde{y}=357293.1-3802.2 \cdot x$ & -0.746 & 0.56 & 23.8 & 0.0001 \\
\hline
\end{tabular}

The received data allows considering the presence of the rigid inverse correlation between the trend of daily revenue and daily average environmental temperature.

10. The forecasting of levels of daily revenue was made in the following sequence:

1) for the each date of forecasted (control) week an expected value of the level of daily average environmental temperature was calculated based on the function of the trend of this index;

2) to receive temperature values according to the regression equation built by using the data of the selection for the previous three weeks, point and interval predictions of the level of the trend component of the value of daily revenue were calculated for each date;

3) a correction of received predicted values of the trend component $(\mathrm{T})$ was carried out by multiplication of the value of the cyclical component $(\mathrm{S})$ corresponding each predicted date in accordance with the model (2). Thus, the recording of variability of the random component $(\mathrm{F})$ was made in the interval prediction calculated on the basis of the standard of the regression model and considered fluctuation of the factor variable, meaning the environmental temperature for the studied three weeks;

4) the error of calculated predictions was estimated by comparison of actual values of daily revenue with the received prediction interval for the control week. In all cases the actual value of revenue was found within the prediction interval that proved stability of the high approximate character of the received regression models and reliability of the results of applied methods of modelling and forecasting.

\section{Conclusion}

Taking into account difficult economic situation in the domestic market of fish and sea products in Russia fish processing enterprises should develop strategies of minimization of expenses during procurement activities - to buy as much as they can sell not only during the shelf life of a product but also so as to sell it quickly without losing consumer appeal and finally, to economize operating assets of the enterprise and provide food safety. In fact, in addition to food availability it's necessary to give consideration to the aspects of its physical and economical availability for the population, also to a criterion of food products safety [10]. The suggested methods of forecasting the levels of daily revenue will allow taking decisions reasonably in order to define planned amount of production and procurement volumes for selling during the short-term prospective period. This is a timely question in the condition of rapidly changing market of consumer goods.

\section{References}

1. V. Selin, Methodological aspects of analyzing and assessing the per capita consumption of fish and seafood in the Russian Federation Economic and Social Changes: Facts, Trends, Forecast 6 (2015)

2. A. Bogachev, Insurance as a way to protect economic interests of aquaculture subjects Agrar. bull. of the Upper Volga region, 2 (2018)

3. B. Il'yasov, V. Martynov, I. Gerasimova, E. Makarova, E. Zakieva, Quality of life: analyzing the impact of factors related to health, based on system and mathematical models Economic and Social Changes: Facts, Trends, Forecast 10, 3 (2017)

4. Z. Ibragimova, The use of economic-organization methods of strategic planning in enterprises of Agro-industrial complex: analyst review Questions of economics structuring 4 (2010)

5. Ol. Cherednichenko, N. Dovgot'ko, N. Yashalova, Sustainable development of the agri-food sector: Russia's priorities and directions to adapt agenda 2030 to Russian conditions Economic and Social Changes: Facts, Trends, Forecast 11, 6 (2018)

6. V. Akimov, R. Durnev, Yu. Sokolov, The influence of climate changes on human health In the book: Protection of population and territories of the Russian Federation in conditions of climate changes (Russian national research institute in regards to civilian protection and emergency situations, Ministry of Emergency Situations of Russia, Moscow, 2016)

7. L. Kormishkina, Ye. Kormishkin, L. Koroleva, D. Koloskov, Recycling in modern Russia: need, challenges, and prospects Economic and Social Changes: Facts, Trends, Forecast 11, 5 (2018)

8. T. Yureneva, O. Barinova, S. Golubeva, Monitoring of the financial condition of agricultural organizations in the Vologda region of Russia in Towards Productive, Sustainable and Resilient Global Agricult. and Food Syst. Conf. proc. (2018)

9. F. Akashi, S. Bai, M. Taqqu, Robust regression on stationary time series: a self-normalized resampling approach J. of Time Ser. Analysis 39, 3 (2018)

10. A. Vasiliev, Fishery complex in the system of Russia's food security Economic and Social Changes: Facts, Trends, Forecast 3 (2009) 